\title{
MRI relaxation parameters predict functional outcome after experimental myocardial infarction
}

\author{
Sebastian M Haberkorn ${ }^{1,2^{*}}$, Christoph Jacoby ${ }^{1}$, Ulrich Flögel ${ }^{2}$ \\ From 19th Annual SCMR Scientific Sessions \\ Los Angeles, CA, USA. 27-30 January 2016
}

\begin{abstract}
Background
Characterization of infarcted myocardial tissue by current cardiovascular magnetic resonance (CMR) methods is predominantly carried out after i.v. application of Gadolinium (Gd)-based contrast agents (CA). However, recent advances in CMR enable the acquisition of parametric maps making use of endogenous tissue properties for diagnostic purposes, which may supersede the need for injection of CA. In the present study, we systematically compared myocardial tissue characterization by Gd-based techniques with intrinsic T1/T2 mapping and their correlation with local cardiac function after experimental myocardial infarction (MI).
\end{abstract}

\section{Methods}

To this end, we used a murine model of MI and monitored the mice over a period of 21 days $(n=9)$. MI was induced by chronic ligation of the distal left anterior descending artery (LAD) and comprehensive CMR was performed at $9.4 \mathrm{~T}$ including cine movies, pre- and postcontrast T1, T2 mapping as well as LGE (Fig. 1). To overcome heart rate associated problems during T1 mapping we used a retrospectively triggered fast low-angle shot sequence with variable flip angle analysis providing constant repetition time and maintaining steady-state conditions. Using this approach high-quality T1 maps could be generated in line with literature data (pre- $1001 \pm 8.6 \mathrm{~ms}$ and post-contrast $289.3 \pm 5.9 \mathrm{~ms}$ ). Extracellular volume (ECV) was calculated from T1 of myocardium and blood pool pre- and post-contrast administration $(32.2 \pm 2.6 \%)$. T2 maps were generated from a gated multi-echo spin-echo sequence (18.8 \pm $2.4 \mathrm{~ms})$. For regional correlation of parametric maps and fractional shortening (FS), all data sets were

'Department of Cardiology, Pneumology and Angiology, University Hospital Duesseldorf, Duesseldorf, Germany

Full list of author information is available at the end of the article analyzed over 160 sectors covering the entire left ventricle (LV) (Fig. 2).

\section{Results}

Longitudinal analysis of mice after MI revealed substantial alterations in MR parameters: At day 1 after MI precontrast T1 and T2 increased up to $1461 \pm 19.2 \mathrm{~ms}$ and $37 \pm 0.5 \mathrm{~ms}$, respectively, while post-contrast $\mathrm{T} 1$ dropped down to $173.9 \pm 5.7 \mathrm{~ms}$ in infarcted myocardium (Fig. 1). Concomitantly, ECV increased to $48.6 \pm 1.9 \%$. To evaluate the prognostic power of the individual measures for functional outcome, data acquired at day 1 were correlated to local wall movement determined at day 21 (Fig. 2). Quantitative analysis of 160 sectors covering the entire LV revealed for all parameters significant agreement with the later outcome (pre-contrast T1 r $=-0.851 ; \mathrm{T} 2 \mathrm{r}=-0.700$; post-contrast T1 $\mathrm{r}=0.606$; ECV $\mathrm{r}=-0.691$; LGE $\mathrm{r}=$ -0.786 , all $p<0.0001)$. Surprisingly, pre-contrast T1 maps on day 1 showed an even better correlation with the FS 21 days after MI than LGE (-0.851 vs. -0.786$)$.

\section{Conclusions}

The present study shows that pre-contrast T1 mapping with variable flip angle analysis carried out 1 day after MI predicts the functional outcome after 21 days at least as reliable as LGE. Compared to the latter technique providing plain signal enhancement, the current approach determines quantitative maps with a large dynamic range, which may pave the way for reliable myocardial tissue characterization without any CA.

\section{Authors' details \\ 'Department of Cardiology, Pneumology and Angiology, University Hospital Duesseldorf, Duesseldorf, Germany. ${ }^{2}$ Molecular Cardiology, University of Duesseldorf, Duesseldorf, Germany.}

Published: 27 January 2016 

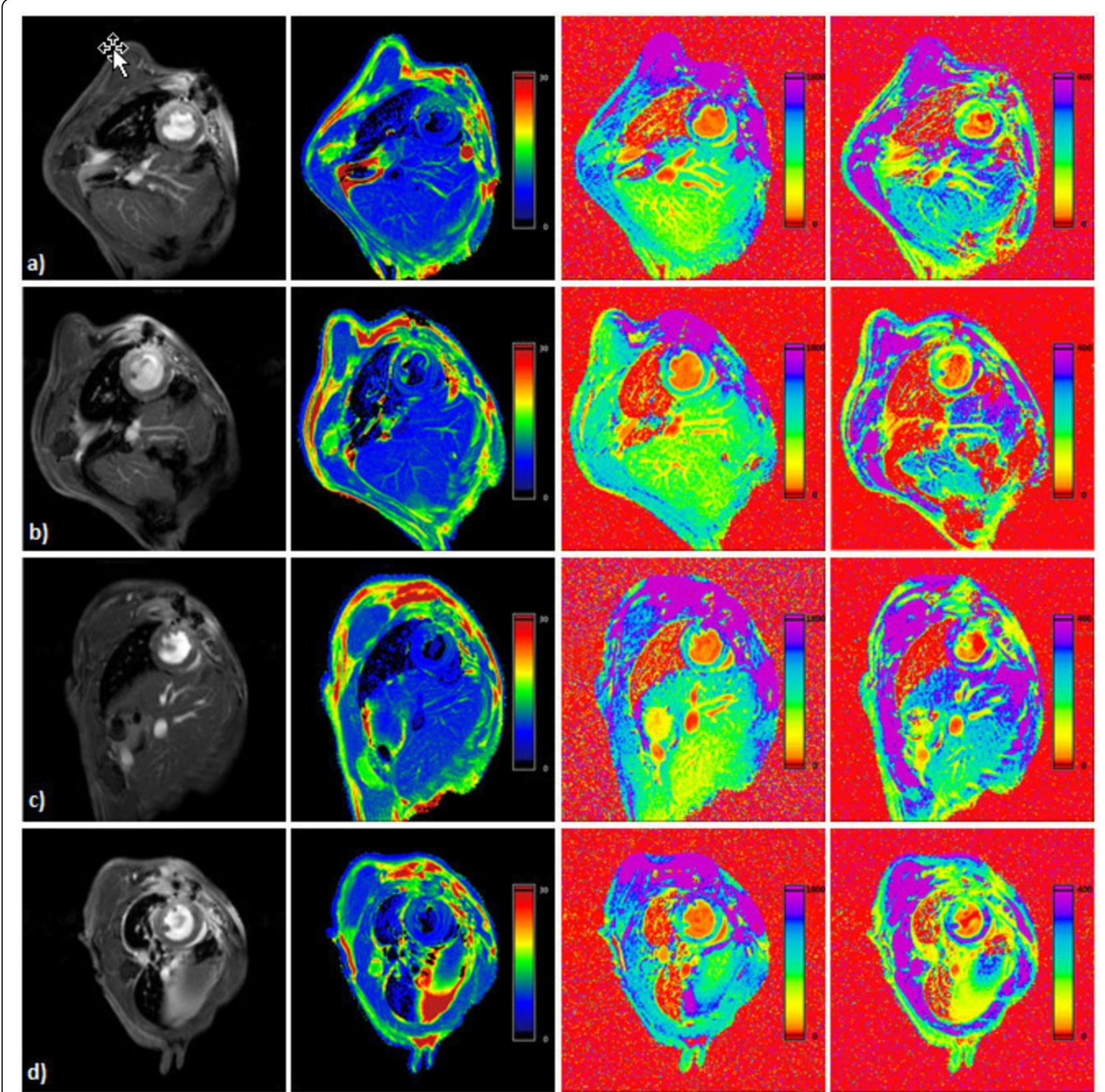

Figure 1

doi:10.1186/1532-429X-18-S1-057

Cite this article as: Haberkorn et al:: MRI relaxation parameters predict functional outcome after experimental myocardial infarction. Journal of Cardiovascular Magnetic Resonance 2016 18(Suppl 1):O57.
Submit your next manuscript to BioMed Central and take full advantage of:

- Convenient online submission

- Thorough peer review

- No space constraints or color figure charges

- Immediate publication on acceptance

- Inclusion in PubMed, CAS, Scopus and Google Scholar

- Research which is freely available for redistribution 

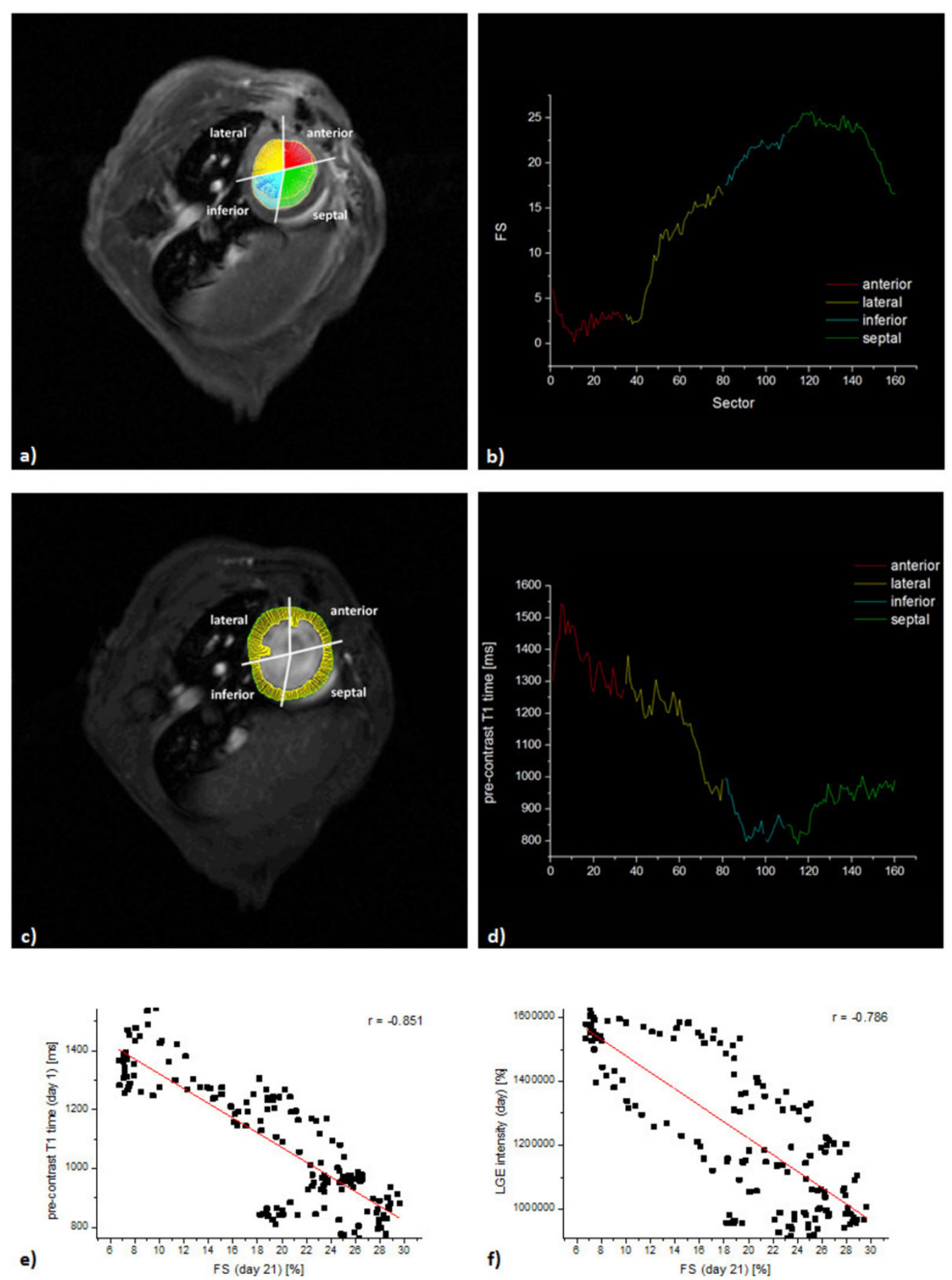

Figure 2 Regional wall movement and parametric analysis over 160 sectors. a) Local wall movement from end-diastole to - systole with color-encoding for the anterior, lateral, inferior, and septal sections of the LV. b) Sectorial analysis of wall movement (give as FS [\%]) with poor contractile function particularly in the anterior wall. c) ROls visualizing endo- and epicardial borders of the LV in end-diastole for pre-contract T1 calculation in each sector. d) regional pre-contrast T1 analysis clearly reveals enhanced T1 values in sectors corresponding to impaired local function in (b). e) Correlation and linear fit of pre-contrast T1 from day 1 with local function (FS) at day 21 ( $r=-0.851, p<0.0001)$. f) Correlation and linear fit of LGE from day 1 with local function (FS) at day 21 ( $r=-0.851, p<0.0001$ ). 